\title{
Streamline Flows for White Matter Fibre Pathway Segmentation in Diffusion MRI
}

\author{
Peter Savadjiev $^{1}$, Jennifer S.W. Campbell ${ }^{1,2}$, G. Bruce Pike ${ }^{2}$, \\ and Kaleem Siddiqi ${ }^{1}$ \\ McGill University, Montréal, QC, Canada \\ ${ }^{1}$ School of Computer Science \& Centre For Intelligent Machines \\ ${ }^{2}$ McConnell Brain Imaging Centre, Montréal Neurological Institute
}

\begin{abstract}
We introduce a fibre tract segmentation algorithm based on the geometric coherence of fibre orientations as indicated by a streamline flow model. The inference of local flow approximations motivates a pairwise consistency measure between fibre ODF maxima. We use this measure in a recursive algorithm to cluster consistent ODF maxima, leading to the segmentation of white matter pathways. The method requires minimal seeding compared to streamline tractography-based methods, and allows multiple tracts to pass through the same voxels. We illustrate the approach with a segmentation of the corpus callosum and one of the cortico-spinal tract, with each example seeded at a single voxel.
\end{abstract}

\section{Introduction and Related Work}

The development of algorithms for automatic white matter fibre tract segmentation in diffusion MRI is of significant interest in the community. A number of methods approach this problem by clustering individual diffusion tensors (DTs) or diffusion orientation distribution functions (ODFs), relying on Euclidean or Riemannian metrics between their shapes, e.g. 81521] for DT data and [1497] for high angular resolution diffusion (HARD) data. Whether implemented using surface evolution techniques [8], spectral clustering 14 15, or various Markov field models 921], these methods assume that along a white matter tract the diffusion ODFs have locally similar shape statistics. It is also often (though not always) assumed that only a single tract passes through any particular location or voxel. These two assumptions can be questioned when: 1) white matter fibre tracts cross, merge or split, 2) an individual ODF reflects multiple fibre populations, 3) partial volume effects occur along a tract, causing the tensor or ODF shape to change drastically and 4) neighbouring but distinct tract systems have regions with ODFs whose shape statistics are very similar.

A second class of automatic segmentation algorithms uses individual streamlines, obtained by a fibre tractography algorithm, followed by a clustering process. For example, Corouge et al. [3] define metrics between individual streamlines, such as the closest point distance, the mean distance, or the Hausdorff distance. Ding et al. [6] propose similar metrics. The Hausdorff distance is also used in [11, within a spectral clustering framework. All these approaches depend 

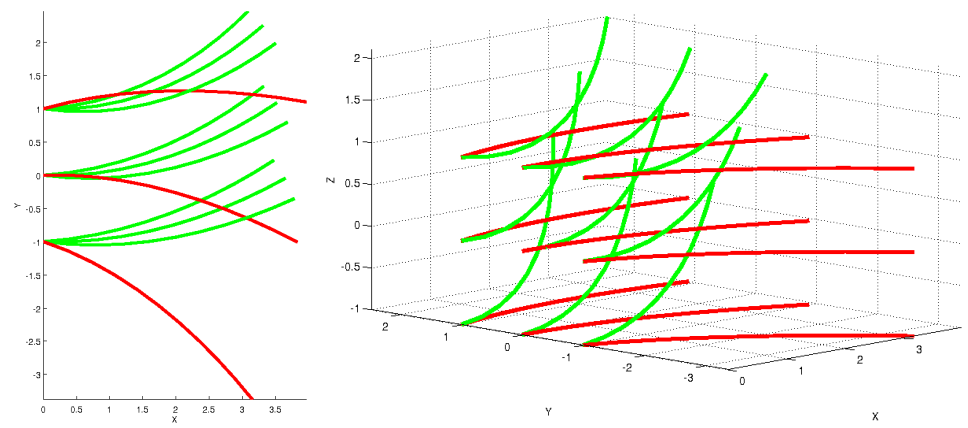

Fig. 1. Two examples of 3D streamline flows generated using equations (2) and (3), each sampled with nine streamlines. Left: An orthographic projection on the XY plane. Right: A 3D view of the flows. The flow parameters are as follows: Green: $K_{T}=0.2$, $K_{N}=0, K_{B}=-0.1, \alpha=1.0$. Red: $K_{T}=-0.2, K_{N}=0.3, K_{B}=0, \alpha=0$.

on the actual tractography algorithm used, and further, they do not explicitly utilize the differential geometry of the 3D space curves that underlie the white matter fibres.

With these limitations in mind, in this paper we model fibre tracts as streamline flows, i.e., sets of dense, approximately parallel curves (locally). Using the differential geometry of these patterns, introduced in [13], and an associated generalized helicoid model, we develop a fibre tract segmentation algorithm which is based on geometrical coherence rather than on ODF shape statistics. The approach takes as its input a volume of fibre ODF data [5. For each fibre ODF maximum at each voxel, a best-fit local model for the underlying streamline flow is then found. Using the inferred streamline flow geometries, a pairwise consistency measure is defined between ODF maxima, leading to a recursive algorithm for clustering them in order to segment white matter fibre tracts. Because the method captures the geometry not only in the tangential (streamline) direction, but also along the normal and bi-normal directions, it requires minimal seeding when compared with methods that rely on streamline tractography (our experiments have used a single voxel seed). It also provides, as a bi-product, local curvature measures of the tracts which can be used to facilitate further analysis.

\section{$23 \mathrm{D}$ Streamline Flow Model}

The streamline flow model we adopt is based on the idea of attaching a frame field to a single streamline, and then examining its "co-variation" in three basis directions 13 . This leads to three curvature functions:

$$
\begin{gathered}
\left\|\kappa_{T}\right\|=\left\|\nabla_{E_{T}} E_{T}\right\|=\left\|\cos \phi d \theta\left(E_{T}\right) E_{N}+d \phi\left(E_{T}\right) E_{B}\right\|, \\
\left\|\kappa_{N}\right\|=\left\|\nabla_{E_{N}} E_{T}\right\|=\left\|\cos \phi d \theta\left(E_{N}\right) E_{N}+d \phi\left(E_{N}\right) E_{B}\right\|, \\
\left\|\kappa_{B}\right\|=\left\|\nabla_{E_{B}} E_{T}\right\|=\left\|\cos \phi d \theta\left(E_{B}\right) E_{N}+d \phi\left(E_{B}\right) E_{B}\right\|,
\end{gathered}
$$


where $\left(E_{T}, E_{N}, E_{B}\right)$ is the Frame Field in spherical coordinates and $\theta$ and $\phi$ describe the orientations of vector $E_{T}$ with respect to a fixed coordinate system. A generalized helicoid model for $\theta$, motivated by minimal surface theory, is (see [13])

$$
\theta(x, y, z)=\tan ^{-1}\left(\frac{K_{T} x+K_{N} y}{1+K_{N} x-K_{T} y}\right)+K_{B} z .
$$

An extended flow model which we adopt in this paper is given by (2), together with the relation

$$
\phi(x, y, z)=\alpha \theta(x, y, z) .
$$

Proposition 1. With $\phi(x, y, z)=\alpha \theta(x, y, z)$, where $\alpha$ is a constant, the scalar parameters $K_{T}, K_{N}, K_{B}$ of the generalized helicoid model (2) and the curvatures (1) of a unique $3 D$ streamline flow are related by:

$$
\begin{aligned}
\kappa_{T}(0,0,0) & =\sqrt{1+\alpha^{2}}\left|K_{T}\right| \\
\kappa_{N}(0,0,0) & =\sqrt{1+\alpha^{2}}\left|K_{N}\right| \\
\kappa_{B}(0,0,0) & =\sqrt{1+\alpha^{2}}\left|K_{B}\right| .
\end{aligned}
$$

Proof. Eq. (44) can be obtained by computing the gradient of $\theta(x, y, z)$ in (2) and noting that at the origin, $\nabla \theta(0,0,0)=\left(K_{T}, K_{N}, K_{B}\right)$. Similarly, given that $\phi(x, y, z)=\alpha \theta(x, y, z), \nabla \phi(0,0,0)=\left(\alpha K_{T}, \alpha K_{N}, \alpha K_{B}\right)$. Since $\phi(0,0,0)=$ $\theta(0,0,0)=0$, this aligns the $\left(E_{T}, E_{N}, E_{B}\right)$ frame at the origin with the global $(x, y, z)$ coordinate system, so that $E_{T}=(1,0,0), E_{N}=(0,1,0)$ and $E_{B}=$ $(0,0,1)$. Eq. (4) follows from substituting the values for $E_{T}, E_{N}, E_{B}, \nabla \theta$ and $\nabla \phi$ into the curvature functions of (10).

\section{Clustering and Fibre Segmentation through Streamline Flow Consistency}

We now develop an algorithm to cluster local fibre orientations to segment fibre tracts. We take as input a dense volume of fibre ODFs extracted from the diffusion signal through a technique such as spherical deconvolution [5]. Each of these fibre ODFs is sampled along a fixed set of predefined unit-length orientations $\lambda$, and the ODF maxima are extracted, as described in Section 4.1 .

\subsection{Co-helicoidity}

We use the generalized helicoid model as an osculating object to provide a local approximation for the streamline flow geometry in a neighborhood around each ODF maximum. We define the following consistency measure $C$ between orientation $\lambda$ at location $i$ and orientation $\lambda^{\prime}$ at location $j$ :

$$
C_{\lambda, i, \lambda^{\prime}, j}=\frac{\left|\left\langle H_{\lambda, i}(j),\left(\lambda^{\prime}, j\right)\right\rangle\right|+\left|\left\langle H_{\lambda^{\prime}, j}(i),(\lambda, i)\right\rangle\right|}{2} .
$$




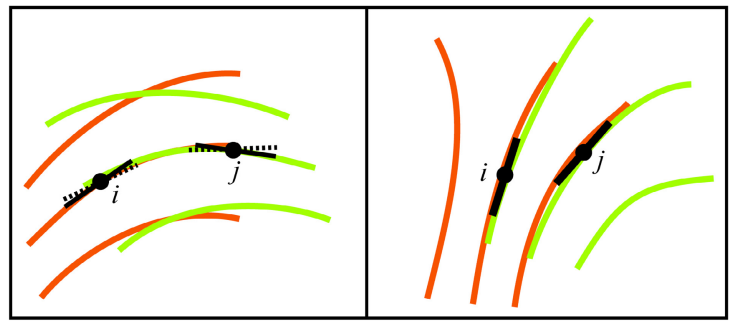

Fig. 2. Two schematic illustrations of the concept of helicoid-based consistency. Left: the orientation at location $i$ (solid black line) is transported to location $j$ along its inferred best-fit helicoid (orange). Similarly, the orientation at location $j$ is transported along its helicoid (green) to location $i$. The transported orientations (dashed black lines) are defined as the tangents to the helicoid at the location of transport. The average angular difference between the transported and local orientations is computed as a measure of consistency. Right: a second transport example, one for which the two helicoids align perfectly at locations $i$ and $j$.

Here |.| denotes absolute value, and $\langle.,$.$\rangle denotes the standard dot product in$ $\mathbb{R}^{3} . H_{\lambda, i}(j)$ denotes the (unit) vector that results from evaluating at location $j$ the generalized helicoid osculating object associated to orientation $\lambda$ at location $i$. Finally, $(\lambda, i)$ denotes orientation $\lambda$ at location $i$ (which is a vector of $(x, y, z)$ coordinates).

Thus, measuring consistency between two orientations defined at two distinct locations involves transporting each orientation in turn along its osculating generalized helicoid to the location of the other orientation, evaluating the resulting angular difference, and then taking the average. Fig. 2 illustrates this concept of transport schematically. We choose this measure of consistency for its simplicity and symmetry. It is a natural extension of the ideas of co-circularity for $2 \mathrm{D}$ planar curves and co-helicity for 3D space curves (see [13] for a review).

\subsection{ODF Clustering and Fibre Tract Segmentation}

We approach the problem of fibre tract segmentation by first inferring the bestfit helicoidal osculating object for each ODF maximum at each location, as described in [13. This operation needs to be carried out only once, offline. Then, starting at a seed voxel selected manually, for each ODF maximum at that voxel, its pairwise compatibility is computed according to (5) with all ODF maxima located within a small neighborhood centered at that voxel (we use a neighbourhood of $3 \times 3 \times 3$ voxels). If the compatibility with a maximum in the neighborhood is higher than a threshold, this maximum is added to the cluster, and the algorithm is recursively called, starting at the newly added maximum. As maxima are added to the cluster, they are marked as 'visited'. Thus, recursion stops when there are no remaining maxima that are both compatible and unmarked. 
As illustrated in Fig. 2, clustering may proceed along flow streamlines as well as across them. Situations in which distinct tract systems 'kiss' each other and form a bottleneck configuration may cause the clustering to progress from one tract into the other and then backtrack along the new tract system. To prevent this, our implementation keeps track of the direction in which clustering of maxima proceeds, starting from the seed voxel, and disallows reversals in that direction. This is achieved by treating ODF maxima as vectors (which have direction), rather than simply as orientations. When a new maximum is added to the cluster, it is given a direction consistent with the direction of cluster expansion. Then, when recursion is called starting at that maximum, it proceeds only in directions consistent with the maximum direction.

\section{Experiments and Results}

\subsection{Methods}

MRI data were acquired for one healthy subject on a Siemens 3T Trio MR scanner (Siemens Medical Systems, Erlangen, Germany) using an 8-channel phasedarray head coil. Diffusion encoding was achieved using a single-shot spin-echo echo planar sequence with twice-refocused balanced diffusion encoding gradients. A dataset designed for high angular resolution reconstruction was acquired with $N=99$ diffusion encoding directions with $b=3000 \mathrm{~s} / \mathrm{mm}^{2}, 10 \mathrm{~T} 2$ weighted images with $b=0 \mathrm{~s} / \mathrm{mm}^{2}, 2 \mathrm{~mm}$ isotropic voxel size, 63 slices, TE $=121 \mathrm{~ms}$, $\mathrm{TR}=11.1 \mathrm{~s}$, and GRAPPA parallel reconstruction with an acceleration factor of 2. The fibre ODF data was estimated using spherical deconvolution as described in [5], with a linear and regularized method performed on the diffusion ODF estimated from q-ball reconstruction. An $L=4$ spherical harmonic basis was used, with regularization parameter $\lambda=0.006$ [4]. The deconvolution kernel was estimated directly from the dataset being used. The maxima of the fibre ODFs, which are assumed to correspond to curve tangents, were then extracted and used in the clustering algorithm described in Section 3.2 A threshold value of $9^{\circ}$ (chosen empirically) was used to determine whether two orientations are consistent according to (5). Voxels where the fractional anisotropy (FA) was less than 0.1 or the mean diffusivity was greater than $10^{-6} \mathrm{~mm}^{2} / \mathrm{ms}$ were excluded.

We compare our segmentation algorithm with conventional streamline fibre tractography with a user-defined, extensive start region of interest (ROI). To do so, a streamline tractography approach robust to complex sub-voxel geometries [12] was used. The ROI was hand drawn on the entire length of the corpus callosum (CC) in the mid sagittal region of the brain. Streamlines were initiated in all voxels of the brain and those that passed through the ROI were retained. Tracking was stopped if the fractional anisotropy (FA) was less than 0.1 or the mean diffusivity was greater than $10^{-6} \mathrm{~mm}^{2} / \mathrm{ms}$. Tracking was seeded on a $3 \times$ $3 \times 3$ grid in each start voxel. 


\subsection{Results}

We present segmentation results for the CC and the cortico-spinal tract (CST). The algorithm of Section 3.2 was initiated in a voxel located in the $\mathrm{CC}$ near the mid-sagittal slice, as well as in a voxel located in the CST. For each starting voxel, a surface encompassing all voxels reached by our recursive segmentation algorithm is shown in Fig. 3. These results are anatomically plausible and demonstrate the recovery of the region common to both tract systems.

To illustrate the sense of a 3D streamline flow, in Fig. 4 we visualize the ODF maxima that are clustered together in a region where the recovered tracts overlap. Again, the recovered flows are anatomically plausible.

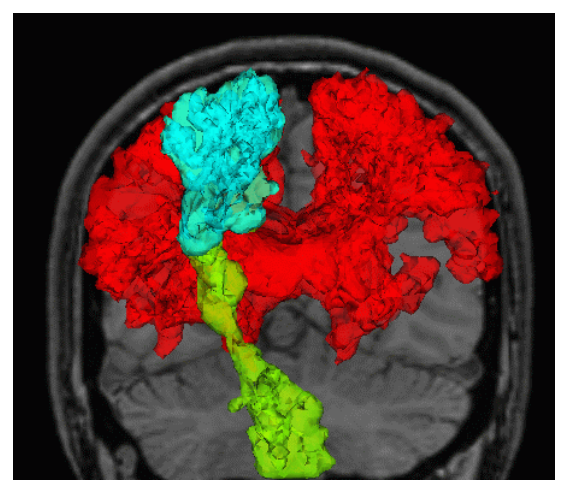

Fig. 3. A segmentation of the corpus callosum (red) and the cortico-spinal tract (green), in a coronal view (seen from the front). The blue region indicates voxels that belong to both of the segmented tracts. The anatomical slice in the background is shown to delineate the head; the recovered clusters are $3 \mathrm{D}$ objects and are not confined to one particular slice.
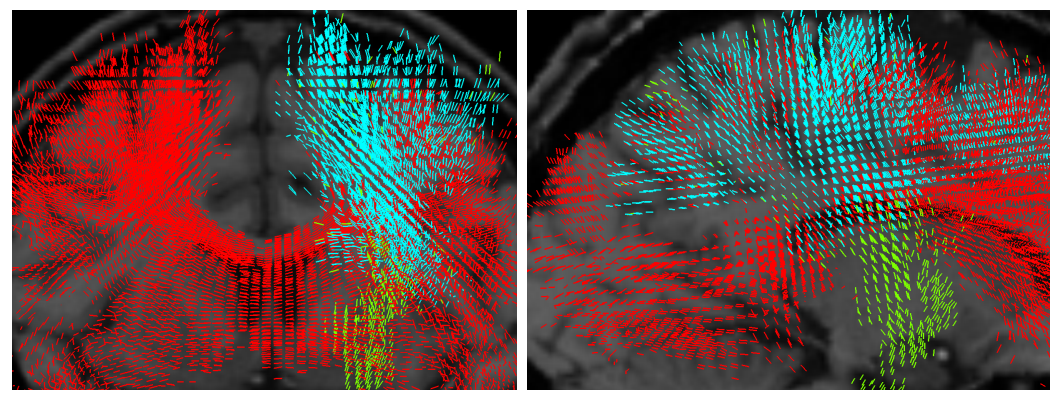

Fig. 4. A zoom-in on the region of cluster overlap as recovered by our algorithm. Left: a coronal projection (seen from behind). Right: a sagittal projection. Both figures visualize the clustered ODF maxima that belong to the corpus callosum (red), the cortico-spinal tract (green), and to both clusters (blue). 

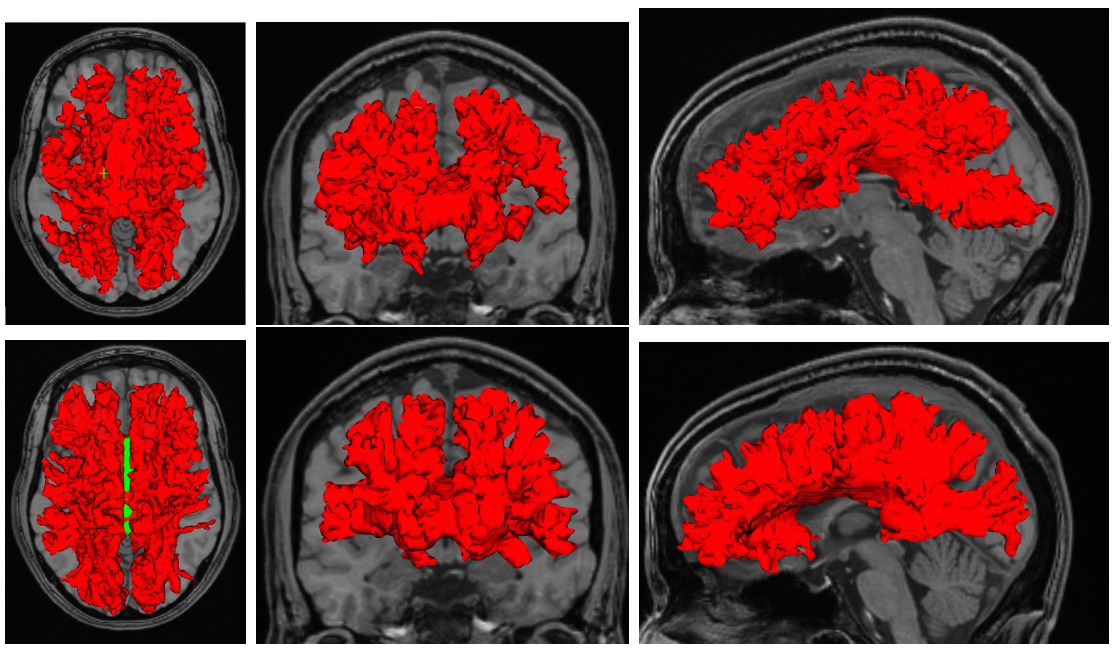

Fig. 5. Top row: corpus callosum segmentation using our method, starting at a seed voxel denoted with a crosshair (left). Bottom row: corpus callosum recovery through streamline tractography initiated in a hand-drawn region (green, left panel).

Fig. 5 compares our segmentation method with conventional streamline tractography on the CC, run as described in Section 4.1. The results are similar in that both methods recover the corona radiata and several lateral projections. However, the streamline tractography method required a hand-drawn initialization region that spans the entire CC (green ROI), whereas our segmentation method is initialized at a single voxel (marked by the green crosshair).

\section{Conclusion}

This article develops a fibre clustering approach based on geometric coherence with respect to a local 3D streamline flow model. The method allows ODF maxima to be clustered not only along individual streamlines, but also across them. For example, when clustering starts with a seed located in the centre of the CC, voxels near the cortex can be reached even if the direct path that connects them to the seed may not be resolvable due to partial volume effects at the crossing with the CST or the superior longitudinal fasciculus. Although the examples in this paper apply the method to fibre ODF data, it is applicable to any type of DT or HARD data. Unlike some surface evolution-based segmentation methods, multiple tracts per location can be handled.

Future work will focus on parameter selection, which is currently done empirically, as well as on a more rigorous validation, done for example through quantitative experiments on a well localized tract. It will also examine whether the segmentation results obtained from a single seed voxel are accurate enough to reduce the need for detailed protocols for the hand-drawing of ROIs [10, which 
would be an advantage in population studies for example. A hand-drawn crosssectional ROI through a tract may be difficult to reproduce and may intersect nearby tracts. Our requirement for a single voxel seed located in a well-defined region of the tract may lead to less user error and better reproducibility. However, a current limitation of our method is that the propagation of support from one streamline to nearby parallel ones can also lead to false positives. We expect that the use of anatomical masks will greatly help to eliminate these. Finally, our co-helicoidity consistency measure can be incorporated as a geometric affinity metric in other existing segmentation frameworks, such as spectral clustering, and can also be used to study fibre tract shape statistics.

Acknowledgments. The authors are grateful to Maxime Descoteaux and Rachid Deriche for help with fibre ODF calculation.

\section{References}

1. Awate, S.P., Zhang, H., Gee, J.C.: A fuzzy, nonparametic segmentation framework for DTI and MRI analysis: with applications to DTI-tract extraction. IEEE Trans. Medical Imaging 26(11), 1525-1536 (2007)

2. Barmpoutis, A., Vemuri, B.C., Shepherd, T.M., Forder, J.R.: Tensor splines for interpolation and approximation of DT-MRI with applications to segmentation of isolated rat hippocampi. IEEE Trans. Medical Imaging 26(11), 1537-1546 (2007)

3. Corouge, I., Gouttard, S., Gerig, G.: Towards a shape model of white matter fiber bundles using diffusion tensor MRI. In: Proc. Intl. Symposium on Biomedical Imaging (ISBI) 2004, vol. 1, pp. 344-347 (2004)

4. Descoteaux, M., Angelino, E., Fitzgibbons, S., Deriche, R.: Regularized, fast, and robust analytical Q-Ball imaging. Magn. Res. in Medicine 58(3), 497-510 (2007)

5. Descoteaux, M., Deriche, R., Anwander, A.: Deterministic and probabilistic Q-Ball tractography: from diffusion to sharp fiber distributions. Technical Report 6273, INRIA Sophia Antipolis (July 2007)

6. Ding, Z., Gore, J.C., Anderson, A.W.: Classification and quantification of neuronal fiber pathways using diffusion tensor MRI. Magn. Res. in Medicine 49, 716-721 (2003)

7. Jonasson, L., Bresson, X., Thiran, J.-P., Wedeen, V.J., Hagmann, P.: Representing diffusion MRI in 5-D simplifies regularization and segmentation of white matter tracts. IEEE Trans. Medical Imaging 26(11), 1547-1554 (2007)

8. Lenglet, C., Rousson, M., Deriche, R.: DTI segmentation by statistical surface evolution. IEEE Trans. Medical Imaging 25(6), 685-700 (2006)

9. McGraw, T., Vemuri, B.C., Yezierski, R., Mareci, T.: Segmentation of high angular resolution diffusion MRI modeled as a field of von Mises-Fisher mixtures. In: Leonardis, A., Bischof, H., Pinz, A. (eds.) ECCV 2006. LNCS, vol. 3953, pp. 463-475. Springer, Heidelberg (2006)

10. Mori, S., Wakana, S., Nagae-Poetscher, L.M., van Zijl, P.C.M.: MRI Atlas of Human White Matter. Elsevier, Amsterdam (2005)

11. O'Donnell, L., Westin, C.F.: Automatic Tractography Segmentation Using a Highdimensional White Matter Atlas. IEEE Trans. Medical Imaging 26(11), 1562-1575 (2007) 
12. Savadjiev, P., Campbell, J.S.W., Descoteaux, M., Deriche, R., Pike, G.B., Siddiqi, K.: Labeling of ambiguous sub-voxel fibre bundle configurations in high angular resolution diffusion MRI. NeuroImage 41(1), 58-68 (2008)

13. Savadjiev, P., Zucker, S.W., Siddiqi, K.: On the differential geometry of 3D flow patterns: Generalized helicoids and diffusion MRI analysis. In: Proc. IEEE Intl. Conf. on Computer Vision (ICCV 2007) (2007)

14. Wassermann, D., Descoteaux, M., Deriche, R.: Diffusion maps clustering for magnetic resonance Q-Ball imaging segmentation. Intl. Journal of Biomedical Imaging (2008); Article ID 526906, doi:10.1155/2008/526906

15. Ziyan, U., Tuch, D., Westin, C.F.: Segmentation of thalamic nuclei from DTI using spectral clustering. In: Larsen, R., Nielsen, M., Sporring, J. (eds.) MICCAI 2006. LNCS, vol. 4191, pp. 807-814. Springer, Heidelberg (2006) 